\section{TIEMPO Y SUCESIÓN ECOLÓGICA EN RAMÓN MARGALEF ${ }^{1}$}

\author{
Esteban Vargas Abarzúa \\ Doctor en Filosofía \\ Pontificia Universidad Católica de Chile \\ Profesor de Filosofía \\ Pontificia Universidad Católica de Valparaíso, Chile \\ esteban.vargas@ucv.cl
}

Luis Zúñiga Molinier

Doctor en Biología Universidad Central de Barcelona Profesor del Instituto de Biología Pontificia Universidad Católica de Valparaíso, Chile

lzuniga@ucv.cl.

\begin{abstract}
In this paper we attempt to think about the time in the Margalef's ecological succession theory. In the classical model of ecological succession, populations succeed each other. Each phases of that succession "as a temporary phase" is merely juxtaposed with the other. It is the clock time, time like "measure" of the clock. Time here is absolute, independent of the process like in Newton. This does not happen in the model of Margalef. He thinks the ecosystem like a process in which energy is transfer and information acumulated. Thus, in the dynamic process of ecosystem appears various phases determined by the intrinsic dynamics of the system. Each ecosystem has its own time.
\end{abstract}

KEY WORDS: Time; ecological succession; ecosystem; information; Margalef.

\section{INTRODUCCIÓN}

Ramón Margalef (1919-2004) fue un afamado ecólogo español que intentó pensar la ecología desde la termodinámica y la teoría de la información. Desde este punto de vista pudo repensar el concepto de espacio y de tiempo como genuinos conceptos ecológicos. Ya desde Newton (1997, 32-33) se concibió el espacio y el tiempo como algo absoluto, independiente de los cuerpos. Si pudiéramos quitar, por ejemplo, todos los cuerpos que existen en una habitación, se piensa que nos quedaríamos, entonces, sólo con el tiempo y el espacio. Ésta es, un poco, la intuición que se tiene cuando se piensa en estos temas. Desde luego, la física, desde los tiempos de Einstein, ha repensado estos conceptos, pero hay que meditar si estas nuevas concepciones han llegado a la biología $y$, en especial, a la ecología. Margalef ha meditado todo esto llegando a

\section{TIME AND ECOLOGICAL SUCCESSION IN RAMON MARGALEF'S THOUGHT}

RESUMEN: En este trabajo intentamos meditar la concepción de tiempo en la teoría de sucesión ecológica de Margalef. En un modelo clásico de sucesión ecológica, las poblaciones se suceden unas a otras. Cada fase de esa sucesión "en tanto fase temporal" está meramente yuxtapuesta a las demás fases. Es el tiempo cronométrico, el tiempo como "medida" del reloj. El tiempo es aquí algo absoluto, independiente del proceso como en Newton. Esto no ocurre en el modelo de Margalef. Él piensa el ecosistema como un proceso de transferencia de energía y acumulación de información. Por esto, en el proceso dinámico del ecosistema existen diversas fases determinadas por la dinámica intrínseca del sistema. Cada ecosistema posee así su tiempo propio.

PALABRAS CLAVES: Tiempo; sucesión ecológica; ecosistema; información; Margalef.

una concepción del tiempo como una especie de variable "dependiente" de la dinámica del ecosistema. En cierta forma, se podría decir que Margalef es el Einstein de la ecología. Su concepción del espacio ecológico la expresa ampliamente en sus textos, pero no así la del tiempo. Por ello, nos proponemos en este escrito explicitar tal concepción. Para desarrollar este tema debemos realizar, en primer lugar, una breve historia de la ecología hasta la época de Margalef. Esto permitirá situarnos en el contexto en que medita el científico español. En segundo lugar, hay que estudiar cuál es la "entidad" o sistema que estudia la ecología y de la cual decimos que el tiempo es una propiedad suya. Luego, en tercer lugar, veremos su dinámica (la llamada sucesión ecológica) y, en cuarto lugar, su carácter temporal, que es el tema que nos importa. Finalmente, a modo de conclusión, presentaremos brevemente algunos problemas del pensamiento ecológico de Margalef. 


\section{Breve historia de la ecología}

Antes de aclarar, entonces, el pensamiento de Margalef convendría realizar brevemente una pequeña historia de la ecología, es decir, de aquello que estudia, atendido esto principalmente desde su aspecto dinámico. La palabra "ecologia" parece haber sido usada por primera vez con claridad por Ernst Haeckel quien la define asi: "Por ecología entendemos la ciencia de las relaciones de los organismos con el mundo exterior, en el que podemos reconocer de una forma amplia los factores de la 'lucha por la existencia'" (1866, II, 286, citado en Deléage, 1993, 73). Para Haeckel, entonces, la ecología estudia las "relaciones" que afectan la lucha por la existencia de los organismos, ya sean éstas inorgánicas (luz, temperatura, etc.) como orgánicas (otros organismos). Es una definición que, pensada del modo correcto, se centra más bien en el organismo que en otros niveles de organización. Hay que hacer notar que la palabra "eco-logía" significa etimológicamente el "estudio de la casa" donde "casa" parece denotar el "medio", el "ambiente" donde el organismo se desenvuelve y que, además, posibilita su existencia. La casa entendida como ambiente es precisamente el "espacio de interacción" de organismos y factores inorgánicos, el "lugar" donde ocurren las interacciones. Es algo que nos recuerda un poco el concepto de espacio absoluto de Newton. Aunque, probablemente, Haeckel no pensó así explícitamente esta etimología, tal concepto se ajusta, en cierto modo, a lo que se quería expresar.

Años después, la ecología comienza a centrarse en otros niveles de organización como poblaciones y comunidades. Así, por ejemplo, Frederic Clements piensa la comunidad vegetal como un tipo de organismo (o superorganismo) que como proceso (formación) se asemeja al desarrollo de un individuo. Así, nos dice:

"The developmental study of vegetation necessarily rests upon the assumption that the unit or climax formation is an organic entity. As an organism the formation arises, grows, matures, and dies [...] The life history of a formation is a complex but definite process, comparable in its chief features with the life history of an individual plant" $(1916,3)$.

La comunidad vegetal, compuesta de poblaciones, es pensada por Clements desde un "lugar" donde habita, para desde allí intentar meditar el orden en que se "suceden" tales poblaciones. Este orden de sucesión no es para él un proceso aleatorio, sino que sigue una cierta dinámica intrínseca (como un organismo) y que culmina en la madurez o climax, la cual es dependiente del clima donde se ubica. Así, por ejemplo, a un lugar llegan primero unas plantas, como las hierbas salvajes, a las que "suceden" otras de raíces más estructuradas, etc. Se establecen, entonces, "fases" ordenadas de sucesión que desembocan en un clímax único. A esta concepción se opone Gleason. Para él, la comunidad no es un superorganismo, sino una sumatoria de especies e individuos que cohabitan en un espacio común. Por ello, su concepción es, como él la llama, "individualista". Así, nos la explica: "The individualistic concept is totally at variance with the idea that the association is an organism, represented by many individuals, and also does not admit an analogy or homology between the species and the association" $(1939,108)$. De esta concepción se sigue que la sucesión es, por tanto, aleatoria o estocástica en el sentido de no estar determinada intrínsecamente como si fuera un superorganismo. No hay fases intrínsecamente ordenadas hacia un clímax único. La sucesión es aquí mero cambio. Sea como fuere esto, sucesión es el cambio en un lugar de unas poblaciones por otras, ya sea que este cambio esté determinado intrínsecamente (Clements) o no (Gleason). Pero hay otras opciones de pensarlo. Tansley mantiene, al igual que Clements, que el concepto de sucesión "involves not merely change, but the recognition of a sequence of phases" $(1935,286)$. Sin embargo, para él, el hecho de pensar la sucesión sólo desde una comunidad entendida como superorganismo complica la explicación de la sucesión. Hay que pensar más bien que la unidad sujeta a sucesión es un "sistema físico" constituido por los componentes biótico y abiótico. Así, nace precisamente el concepto de "ecosistema". Tansley nos lo explica asi:

"But the more fundamental conception is, as it seems to me, the whole system (in the sense of physics), including not only the organism-complex, but also the whole complex of physical factors forming what we call the environment of the biome -the habitat factors in the widest sense [...] It is the systems so formed which, from the point of view of the ecologist, are the basic units of nature on the face of the earth. Our natural human prejudices force us to consider the organisms (in the sense of the biologist) as the most important parts of these systems, but certainly the inorganic 'factors' are also parts [...] These ecosystems, as we may call them, are of the most various kinds and sizes. They form 
one category of the multitudinous physical systems of the universe, which range from the universe as a whole down to the atom" $(1935,299)$.

La relación extrínseca de Haeckel es pensada ahora intrínsecamente por Tansley, una relación de momentos o partes que constituyen un verdadero sistema físico al modo como lo son los átomos. Es justamente un "eco-sistema". Pero ¿de qué relación se trata? La ecología fue comprendiendo poco a poco que las "relaciones intrinsecas" son, en rigor, las transferencias energético-materiales. Aquí, puede destacarse el trabajo de Lindeman quien recibió el influjo de Elton y principalmente de su maestro Hutchinson. Lindeman definió el ecosistema de la siguiente manera: "The ecosystem may be formally defined as the system composed of physical-chemical-biological processes active within a space-time unit of any magnitude, i.e. the biotic community plus its abiotic environment" (1942, 400). Lindeman, siguiendo a Tansley, concibe el ecosistema como un sistema en el cual sus procesos son "trófico-dinámicos", donde trófico quiere decir alimento. Los organismos se comen unos a otros y con ello se traspasan energía. Por ello, nos dice: "The basic process in trophic dynamics is the transfer of energy from one part of the ecosystem to another" $(1942,400)$. La energía que proviene de la luz solar es capturada por las plantas y luego es transferida a los consumidores, etc. Esto establece niveles tróficos cuya transferencia energética comienza a ser conocida poco a poco a través de la medición de una serie de indicadores como productividad, asimilación, respiración, eficiencia, etc. Estudiando estos indicadores en algunos ecosistemas, Lindeman pudo encontrar algunos patrones en el flujo de un nivel a otro como, por ejemplo, la pérdida progresiva de energía (debida a respiración) o el aumento de la eficiencia. Esto último nos lo explica asi: "These progressively increasing efficiencies may well represent a fundamental trophic principle, namely, that the consumers at progressively higher levels in the food cycle are progressively more efficient in the use of their food supply" $(1942,407)$. Por último, Lindeman intenta pensar todos estos indicadores tróficos en la "dinámica" del ecosistema, en la sucesión ecológica. Así, nos dice: "From the trophic-dynamic viewpoint, succession is the process of development in an ecosystem, brought about primarily by the effects of the organisms on the environment and upon each other, towards a relatively stable condition of equilibrium" (1942, 409). Desde este aspecto "trófico- dinámico" Lindeman cree descubrir algunos patrones como el aumento de la productividad de un lago en el tiempo que va de oligotrófico a eutrófico (algo que después va a discutir Margalef) ocurriendo, finalmente, un decaimiento de la productividad (lago senescente). Como quiera que sea, la sucesión no es ahora sólo el reemplazo de unas poblaciones por otras, sino, radicalmente, una variación de las relaciones tróficas mismas que hace que el lago, como ecosistema, pase por distintas fases. Es esta línea de investigación la que van a seguir, por ejemplo, los hermanos H. T. y E. P. Odum y el mismo Margalef. Esta historia que presentamos es bastante incompleta, pero nos muestra, a grandes rasgos, dos etapas de la ecología; el estudio de los organismos y su relación con el ambiente, y el estudio trófico-dinámico de las relaciones entre los componentes biótico y abiótico del ecosistema. Con esto en mente, veamos ahora el pensamiento de Margalef.

\section{El ecosistema según Margalef}

Como señalábamos antes, Margalef se coloca en la tradición que piensa que la ecología no estudia sólo los organismos (no es una rama de la biología) ni ninguna de las supuestas unidades supraorganísmicas "por separado" (poblaciones, comunidades, etc.), sino que estudia un nivel de organización determinado por las "relaciones intrínsecas" entre los momentos biótico y abiótico que constituyen el sistema llamado ecosistema. Así, Margalef dice: "La ecología, a mi entender, es el estudio de los sistemas a un nivel en el cual los individuos $u$ organismos completos pueden ser considerados elementos de interacción, ya sea entre ellos, ya sea con matriz ambiental laxamente organizada. Los sistemas, a este nivel, se denominan ecosistemas y la ecología, evidentemente, es la biología de los ecosistemas" $(1978,10)$. Ahora bien, esta interacción, como se ha visto desde los tiempos de Lindeman, es el intercambio de materia-energía. La ciencia física que estudia precisamente estos intercambios, desde el punto de vista energético, es la termodinámica. De este modo, podría pensarse que la ecología es, en cierta forma, termodinámica de ecosistemas. Sin embargo, la termodinámica estudiaba, especialmente por aquel entonces, sistemas cerrados, cosa muy distinta a lo que parece suceder en los sistemas vivos y en los ecosistemas. Por ello, Margalef ocupa, como modelo teórico, la termodinámica y la teoría de la información que 
le ayudan a entender la dinámica de los sistemas termodinámicos abiertos. Así, sobre la vida, Margalef nos dice:

"Si tuviera ahora mismo que dar una definición a bote pronto de la vida, diría que es una actividad sistémica que cataliza la conversión de entropía en información. Me explicaré. La energía no puede utilizarse dos veces seguidas de la misma manera; en otros términos, se degrada. Esta degradación se asocia con el aumento del valor de una función calculada que se llama entropía. Ahora bien, mientras se producen esos cambios, la energía ha interactuado con la materia, de suerte que lo que hemos Ilamado degradación de la energía aparece reflejado en otro lado, seguramente contabilizable también, a través de la adquisición de complejidad por parte de los sistemas materiales en los que dichos cambios de energía se suceden" (1992, XI).

Los seres vivos utilizan la energía para formar biomasa, para formar más de sí mismos. Son estructuras autopoiéticas. La energía utilizada se degrada (aumenta la entropía), pero queda algo de aquélla en las estructuras materiales formadas (por ejemplo, en los enlaces químicos). Este proceso restringe las posibilidades futuras. Es en este sentido muy amplio en el que Margalef señala que el sistema vivo acumula información. Por ello, nos dice: "Información es lo que caracteriza y distingue una ordenación o arreglo particular de elementos entre un gran número de posibles ordenamientos" $(1991,90)$.

Por otra parte, Margalef intenta pensar esta misma conversión de entropía en información en los ecosistemas. Así, sobre el ecosistema, nos dice:

"La energía que entra en el sistema constituye la producción primaria, a nivel de los vegetales, y esta energía progresivamente aminorada -aproximadamente a una décima parte en cada nivel- pasa del nivel trófico de los productores primarios a otros niveles tróficos constituidos por fitófagos, zoófagos de primer grado, zoófagos de segundo grado, etc., y descomponedores o reductores. La energía forma un ciclo abierto, pues, finalmente, se disipa -respiración- de forma no recuperable [...] Estas relaciones se entienden mejor considerando al ecosistema como un sistema cibernético. Entre sus propiedades figura la de ser capaz de incrementar su organización, de manera automática, y esta tendencia a alcanzar estados cada vez más maduros, constituye la base de la sucesión ecológica" (1967, 383-384).
Margalef, como Lindeman, ve en el flujo energético de un nivel trófico al siguiente, una pérdida de energía, pero habría que mirar también una cierta acumulación de la información. La apuesta de Margalef es mirar toda la ecología desde este punto de vista como en la relación depredador-presa, los índices de diversidad, la relación productividad/biomasa, etc. Es desde esta concepción que pretende entender el ecosistema desde un punto de vista dinámico, proceso que Ilama sucesión ecológica. Se podría decir, parafraseando a Lindeman, que su postura es "informático-dinámica". ¿Cómo entiende Margalef la sucesión ecológica? Lo veremos a continuación.

\section{La sucesión ecológica según Margalef}

Margalef, siguiendo cierta tradición, ha puesto esencialmente el tema de la sucesión en el ecosistema y no en los organismos, poblaciones o comunidades por separado. Lo que se sucede o cambia, en rigor, no son las poblaciones en una comunidad, sino la acumulación de la información. Por ello, nos dice: "El proceso de la sucesión es equivalente a un proceso de acumulación de información" (1978, 32). No es que no exista un recambio de especies o poblaciones en una comunidad, pero no ésta la mirada radical del ecólogo. Él intenta tratar de entender la "interacción" que se había venido estudiando en la historia de la ecología, pero desde un enfoque especial. Para entender mejor este punto de vista, hay que tener en cuenta que, para Margalef, hay otros dos grandes procesos que pueden entenderse de esta misma manera. Así, nos dice:

"En biología se pueden reconocer, por lo menos, tres clases de procesos o de secuencias históricas [...] Dichas tres clases de procesos o secuencias temporales son: (1) la vida individual, (2) la sucesión ecológica y (3) la evolución. Las tres se hallan muy relacionadas entre sí y comparten en muchas maneras las formas de adquisición, elaboración y proyección hacia el futuro de información" (1998, 173).

Ya hemos visto, brevemente, que la vida individual podría pensarse como un proceso de acumulación de información. No entremos en ello. Más extraño es pensarlo en la evolución. Aquí, Margalef parece mirar la dinámica de las especies (especiación) como un proceso de complejización. Es lo que explicaria, tal vez, los distintos tiempos geoló- 
gicos de cambio de las especies (bruscos al comienzo y después estables, según como lo han señalado Eldredge y Gould). No es un tema que podamos desarrollar aquí. Ahora bien, hay que meditar esta misma idea en el ecosistema. Margalef ha intentado buscar indicadores de esta creciente acumulación de información y complejidad. Walker ha resumido esas propuestas de Margalef en siete pasos:

"1. La biomasa y la producción incrementan a lo largo de la sucesión, pero con tasas diferentes, lo que supone una disminución del cociente entre producción primaria neta y biomasa.

2. La masa de heterótrofos incrementa con relación a la biomasa total.

3. La longitud de las cadenas tróficas incrementa.

4. El número de especies incrementa y, a menudo, también la diversidad.

5. El reciclado interno de nutrientes incrementa y la tasa de renovación disminuye.

6. Los mecanismos de homeostasis devienen más efectivos, en parte gracias a una mayor longevidad de los organismos.

7. La sucesión es, por tanto, un proceso de auto-organización" $(2005,3)$.

Todas estas propuestas responden a una consecuencia del modelo de sucesión como acumulación de información. Para entender esto, estudiemos la primera propuesta, la que Margalef considera que probablemente describe mejor la sucesión. Así, él nos dice:

"El mejor descriptor del estado o momento histórico de la sucesión es, probablemente, el cociente entre la producción primaria total o bruta y la biomasa total del ecosistema, incluyendo bacterias, hongos y animales. Este cociente disminuye a lo largo de la sucesión normal; primero, rápidamente, más tarde, de manera más pausada. Su valor puede servir de criterio para ordenar las distintas etapas de una sucesión, y aun distintos ecosistemas, en una escala común de madurez" $(1992,233)$.

La producción primaria total $(\mathrm{P})$ del ecosistema nos muestra la cantidad de energía fijada por unidad de tiempo. Esta energía queda fijada en la biomasa (B). A mayor producción habrá mayor cantidad de biomasa. El problema es que ambas medidas no crecen proporcionalmente en el ecosistema. La biomasa se acumula en mayor grado, hay cada vez menos disipación de energía. Eso se vería reflejado en que el coeficiente P/B disminuye. Este coeficiente es, en el fondo, el inverso del tiempo ( $1 / \mathrm{t})$. Visto desde el punto de vista informacional lo que ocurre es que, a medida que ingresa la energía, se va acumulando información en la biomasa. Esto limita las posibilidades futuras. Aumenta la eficiencia, cada vez se disipa menos energía. En función de esto, pueden leerse las otras propuestas de Margalef. Por ejemplo, la masa relativa de los heterótrofos aumenta, pues, estos organismos son más complejos y acumulan más información. Del mismo modo hay que entender el largo de las cadenas tróficas. Mientras más largas hay menos disipación de energía, cada vez se aprovecha mejor la energía acumulada en la biomasa. Ahora bien, hay que señalar que, para Margalef, toda esta sucesión no culmina en un clímax como punto último de equilibrio. Más bien, tiende a concebir el clímax como un punto ideal asintótico. Como veremos, el proceso ecológico puede tener reversiones producidas por las catástrofes.

\section{Ecosistema y tiempo en Margalef}

Nos queda ahora ver qué es el tiempo para Margalef. Es un tema que no ha hecho tan explícito en sus libros y trabajos. La ocasión de pensar este tema en Margalef nos permite vislumbrar la posibilidad de atender a un tiempo propiamente ecológico, distinto del tiempo más afín a la física y la biología, el tiempo "cronométrico". Por ello, antes de entrar en el tema convendría distinguir estos dos tipos de tiempo. Por una parte, hay que atender al tiempo "cronométrico" que es el tiempo entendido como "medida" del reloj. En este caso se trata de poner en relación de simultaneidad dos procesos físicos en tanto sucesivos, como ya lo señaló Einstein $(1984,24-26)$. El primer proceso es el del reloj mismo. Un "buen" reloj debe poseer diversas fases sucesivas, pero éstas deben ser "equidistantes", es decir, por ejemplo, que la "distancia temporal" entre el tercer y cuarto segundo sea la misma que entre el cuarto y el quinto. El segundo proceso es el ente físico a medir. Lo que hacemos es poner en relación de simultaneidad las fases del proceso del reloj con las del ente físico en cuestión. Lo medido es justamente el tiempo cronométrico (una hora, diez minutos, etc.).

Ahora bien, todo esto puede usarse para medir el tiempo cronométrico de los procesos biológicos, pues, éstos 
son, ante todo, procesos físicos. Así, por ejemplo, podemos medir la llamada "edad cronológica" (cronométrica) de una persona en años. Pero es posible pensar el tiempo de otra manera. En un organismo individual, las fases de su desarrollo están determinadas por el "proceso mismo" y no en función de las fases de un reloj. Así, por ejemplo, si tenemos varias fases en el organismo (a una fase "A" le sigue una "B", a ésta una " $C$ ", etc.), la determinación de cada fase no se establece por su distancia cronométrica (como, por ejemplo, si se dijera que el proceso biológico se divide intrínsecamente en fases de intervalos de una hora), sino por las determinadas "cualidades" que presenta la dinámica del sistema, por ejemplo, las fases del desarrollo pre natal (segmentación, gastrulación, etc.) o post natal (infancia, adultez, vejez, etc.). Estas fases constituyen la "edad biológica", algo muy distinto del mero número que mide la "edad cronológica". La edad como fase temporal cualitativa merecería tener su lugar en la concepción del tiempo ${ }^{2}$. Constituye un "tiempo biológico cualitativo" (en este caso, la edad biológica) que es "dependiente" del proceso de desarrollo del organismo. El tiempo biológico como edad no es una variable independiente que corre por fuera de los procesos del organismo, sino que es dependiente de éstos. Hay que intentar superar aquí la concepción del tiempo absoluto de Newton.

Considerando esto, se podría intentar pensar un tiempo propio en la sucesión ecológica. Desde luego, este posible tiempo ecológico no excluye el tiempo cronométrico ya que los procesos ecológicos son, ante todo, procesos físicos de sucesión. El problema es que el número que expresa la medida temporal de un proceso (meses, años, etc.) no expresa nada sobre la dinámica intrínseca de tal proceso. Hay que averiguar, entonces, si en el cambio ecológico existe un proceso con una dinámica interna propia. Si la hay, la medida de tiempo cronométrica es insuficiente para expresarla, se requiere de una determinación temporal cualitativa de las fases. Esto es precisamente lo que hay que averiguar en el problema del tiempo de la sucesión ecológica. Ahora bien, para aclarar mejor este problema, coloquemos un ejemplo imaginario. Supongamos que se crean al mismo tiempo cronométrico, dos lagos. Ambos tendrian siempre la misma "edad cronológica" en años. Introduzcamos en ellos diversos organismos, relativamente distintos para cada lago. ¿Es posible establecer en ellos diversas fases procesuales que nos permitan establecer diferentes "edades ecológicas", si se nos permite la expre- sión? ¿0 es el ecosistema o el lugar geográfico, un mero receptáculo donde meramente se suceden las poblaciones o especies en una comunidad? Dicho de otra manera, el tiempo de la sucesión ecológica, ¿es acaso el tiempo del reloj que pasa imperturbable por sobre las poblaciones, comunidades y ecosistemas? Nosotros creemos que Margalef, aunque no tan explícitamente, piensa un tiempo ecológico propio, distinto del mero tiempo cronométrico. Para aclarar esto, hay que recordar que, para Margalef, existen tres grandes procesos que él Ilama históricos ${ }^{3}$, pero que es mejor llamar temporales. Esos procesos son el desarrollo de la vida individual, la especiación (evolución) y la sucesión ecológica. Los tres se caracterizan por una acumulación de información. Los tres procesos constituirían tres tipos de tiempo biológico. Dejemos de lado los dos primeros procesos, no es nuestro tema aquí. ¿De qué manera se entiende aquí el tiempo en la sucesión ecológica?

Ante todo, hay que tomar en cuenta que, por ejemplo, en un lago o bosque, podrían establecerse diversas fases temporales determinadas por el proceso mismo del ecosistema. Esas fases pueden establecerse porque cada fase del proceso está restringiendo las posibilidades del siguiente momento. Las fases ecológicas no son meramente sucesivas como las fases de un reloj. Para entender todo esto hay que intentar responder a dos preguntas: ¿cómo se determinan las fases de un ecosistema? y ¿cómo cada etapa determina la siguiente? Veámoslo separadamente.

1. Por una parte, hay que ver cómo establecer las fases. Para que exista un tiempo no cronométrico estas fases no pueden establecerse con un reloj. No puede decirse, por ejemplo, que el lago tiene tantos años, porque esa medida es extrínseca al proceso. En el concepto cronométrico de tiempo, las fases temporales son establecidas extrínsecamente $y$, por tanto, son independientes del proceso ecológico mismo. Lo que suceda en el ecosistema no hace variar, por ejemplo, los minutos, meses, etc., que establece un reloj. El tiempo cronométrico es así una variable independiente. Para vislumbrar un tiempo ecológico no cronométrico hay que atender a los medidores de la acumulación de información o, si se quiere, de complejidad del propio ecosistema. Por ejemplo, puede usarse el indicador $\mathrm{P} / \mathrm{B}$ que es, como vimos, el más significativo para Margalef. Ese indicador, u otros que se usen, pueden indicarnos una especie de fase de juventud o madu- 
rez ecológica de un lago. Así, recordando el ejemplo ideal expresado más arriba, es posible que dos lagos, entendidos como ecosistemas, pudieran tener la misma "edad cronológica", pero distinta "edad ecológica". Como cada ecosistema posee sus propias vicisitudes, cada ecosistema poseería así su propio tiempo ecológico. Nos parece que ése es el concepto de tiempo que se desprende de la teoría ecológica de Margalef. Ahora bien, un ecosistema, ¿cuántas fases posee? Eso no es fácil de precisar. Margalef nos muestra fases "cualitativas" (lago menos maduro, más maduro). Recordemos que Margalef evita hablar de clímax. Pero podría ayudarse esa clasificación de fases con los medidores "cuantitativos" ya señalados como $P / B$ que es el inverso del tiempo. El valor recíproco $B / P$ es ya una medida de tiempo, pero de tiempo cronométrico. Podrian buscarse, tal vez, indicadores más cualitativos. En fin, todo eso son problemas de ecología. Aquí, intentamos comprender cómo podría entenderse un tiempo propiamente ecológico y dependiente de la dinámica del ecosistema.

2. Por otra parte, hay que establecer cómo cada fase determina la siguiente. En una concepción de tiempo "puramente cronométrica" de la sucesión ecológica, cada fase, "en tanto fase temporal", pasa independientemente de la anterior. Su única unidad es el pasar "después de" la fase anterior. Esto no quiere decir que la concepción cronométrica de la sucesión de poblaciones en una comunidad tenga que ser, por ello, aleatoria. Eso es otra cuestión. Porque incluso si se concibe la sucesión como ordenada intrínsecamente, pero se entiende su tiempo como cronométrico, cada fase temporal (horas, meses, etc.), en tanto temporal, es independiente de la fase anterior. La sucesión es, en este concepto cronométrico, sólo un presente que va pasando. El mundo, temporalmente hablando, primero aparece (presente) y luego se hunde en el pasado. El pasado es algo aquí negativo, algo perdido para siempre, recuperado sólo por la memoria humana ${ }^{4}$. Distinto es lo que ocurre, para Margalef, en la sucesión ecológica. Las fases están ligadas unas a otras. Cada momento temporal recoge algo del anterior y delimita las posibilidades del siguiente. Así, Margalef nos dice: "Desarrollo histórico en general y sucesión ecológica son exactamente lo mismo: las etapas posteriores se alimentan de parte del excedente de etapas anteriores: el futuro, eventualmente, se alimentará del presente" $(1991,102)$. El pasado no es aquí algo que "no es", sino que, de algún modo, se conserva en el presente, en sus estructuras materiales. De ahí que el ecólogo pueda investigar en el presente lo que ha ocurrido en el pasado. Del mismo modo, el futuro está determinándose desde el presente. Esto hace que el mundo no sea caótico $y$, por tanto, sea posible la ciencia. Ahora bien, atendamos ahora a la "dirección" de las fases del ecosistema. Margalef nos lo explica así:

"Los cambios ecológicos se pueden clasificar en dos grupos: cambios bruscos, producidos ordinariamente por algún impacto energético procedente del exterior, y cambios lentos y graduales, en el curso de los cuales la energía externa se degrada paulatinamente bajo el control del ecosistema y los elementos internos del ecosistema se van segregando paulatinamente" $(1982,56)$.

Los cambios de una fase a la siguiente son lentos y graduales. Este paso está determinado por una matriz de probabilidades de transición y no por el paso del reloj (cf. Margalef, 1991, 235). Es justo lo que Margalef ha conceptuado como sucesión ecológica. Parecen dirigirse hacia un punto atractor llamado clímax. Independiente de la creencia en este punto (que Margalef más bien piensa como un ideal asintótico) lo cierto es que hay un proceso natural de acumulación de información. Pero también es posible el paso contrario producido por la catástrofe. No es un paso gradual como el dirigido hacia la madurez, sino un paso abrupto e imprevisto. No es un paso hacia el pasado en el sentido del tiempo cronométrico, sino un retroceso en las etapas o fases del tiempo ecológico. Desde luego, Margalef no piensa que se vuelva exactamente a la misma condición anterior, lo que revela que las fases no son algo tan preciso. Pero, en líneas generales, sirven para mostrarnos cómo debe ser pensado el tiempo ecológico. Y esto es precisamente lo que habria que seguir meditando.

\section{Algunos problemas de la teoría ecológica de Margalef}

Hemos visto cómo desde el pensamiento de Margalef puede esbozarse una nueva concepción del tiempo en la sucesión ecológica que no se reduzca al tiempo cro- 
nométrico. El tiempo ecológico es algo dependiente del proceso ecosistémico y no una variable independiente que pasa incólume por sobre los organismos, poblaciones y comunidades. Pero, para que esta concepción pueda dar sus frutos, desde la concepción de Margalef, habria que preguntarse cuáles son algunos de sus supuestos y meditarlos. Es lo que nos gustaria dejar planteado, a modo de conclusión. Ante todo, habría que preguntarse, y no sólo en el caso de Margalef, sino para todos los ecólogos, cómo se nos presenta un ecosistema. ¿Es algo que puede verse, tocarse, etc.? Casi todos los ecólogos dirian que el ecosistema es un modelo, pero todo modelo, para probar su valía, debe basarse en algo percibido. La ecología empezó por estudiar los organismos y cómo el entorno los modifica. Luego, puso el acento en las relaciones tróficodinámicas. La "relación" parece ser algo observable, pero eso no es tan claro en las relaciones ecológicas. Es un problema a meditar. En segundo lugar, hay que pensar el ecosistema como canal de información. Margalef parece tener la "intuición" de que el ecosistema se comporta dinámicamente tal como lo hace un organismo en el cual, cada momento está siendo determinado por el anterior. Esa "intuición" es la que es modelada desde la teoría de la información y la complejidad. Margalef no duda de que la teoría de la información sea sólo un "modelo" que "podria" explicar la sucesión ecológica. El asunto entonces es meditar el rango epistemológico de esa "intuición" ¿Es algo que todos podemos percibir? ¿0 es tan sólo un modelo sacado de la verdadera intuición que es el desarrollo de los organismos o los procesos históricos? En tercer lugar, hay que meditar el significado de los indicadores de sucesión ecológica. ¿Realmente miden los grados de madurez del ecosistema? La ecología de hoy parece meditar la sucesión de forma menos holística. Es algo también a meditar. Por ultimo, hay que seguir pensando el tiempo biológico como algo dependiente del proceso y no al modo de Newton. Esto parece relativamente claro en el tema de la edad en el organismo. Pero ¿y en la sucesión ecológica? Hemos escogido el pensamiento de Margalef porque en él están los cimientos para una posible mejor concepción del tiempo ecológico, a pesar de todos los problemas que puedan existir en su concepción ecológica.

\section{NOTAS}

1 Este trabajo fue posible gracias a un proyecto FONDECYT Chile de Postdoctorado n. 3085042.

2 La edad como estructura temporal propiamente biológica ya ha sido levemente vislumbrada por el filósofo español Zubiri $(2008,254)$. Todo su trabajo sobre el tiempo ha sido profundamente inspirador para nosotros.

3 Zubiri también ha intentado pensar la edad como fase de un proceso histórico $(2006,161)$. Es lo que llama "edad histórica" como cuando se habla de la Edad Media o la Edad de Piedra. Tales edades no son determinadas por el reloj, sino por criterios históricos que buscan determinar las cualidades o fases intrínsecas del proceso. La Historia, entonces, se asemeja mucho a los tres procesos que ha descrito Margalef (vida individual, evolución, sucesión) tanto es así que a los tres los ha llamado procesos históricos. De esto, ¿puede decirse que la Historia sea, desde un cierto aspecto, un proceso de acumulación de información? Es algo a meditar.

4 El tiempo como "medida de la sucesión", cuyos antecedentes provienen de Aristóteles, ya ha sido extensamente discutido por la talla de filósofos como Bergson, Heidegger, Zubiri y Ricoeur. El tiempo de la conciencia, por ejemplo, del que tratan obras como la de Husserl y James, no tiene este carácter meramente sucesivo. Pero otra cosa es pensarlo en la tal vez mal llamada "sucesión" ecológica. Es lo que intentamos meditar aquí desde el pensamiento de Margalef.
Recibido: 28 de octubre de 2009

Aceptado: 15 de diciembre de 2009 


\section{BIBLIOGRAFÍA}

Clements, F. (1916): Plant Succession, Washington, Washington Press.

Deléage, J. (1993): Historia de la ecología, Barcelona, Icaria.

Einstein, A. (1984): Sobre la teoría de la relatividad especial y general, Madrid, Alianza.

Gleason, H. (1939): "The individualistic Concept of the Plan Association", en American Midland Naturalist, vol. 21, n. ${ }^{\circ} 1,92-110$.

Haeckel, E. (1866): Generelle Morphologie der Organismen, II, Berlin, Reimer.

Lindeman, R. (1942): "The Trophic-Dynamic Aspect of Ecology", en Ecology, 23, 399-417.
Margalef, R. (1967): "El ecosistema", en Ecología Marina, vol. 12, Fundación La Salle, Caracas, 377-453.

- (1978): Perspectivas de la teoría ecológica, Barcelona, Blume.

- $\quad$ (1982): "El ritmo de la evolución en el marco cambiante de la biosfera", Conferencia a la Universitat de Barcelona el dia 19 d'abril de 1982, en Darwin a Barcelona, 1984, Barcelona, Promociones Publicaciones Universitarias: 51-59.

- (1991): Teoría de los sistemas ecológicos, Barcelona, Publicacions de la Universitat de Barcelona.

- (1992): Planeta azul: Planeta verde, Barcelona, Biblioteca Scientific American, Prensa Científica.

- (1998): "Progreso: una valoración subjetiva entusiasta de casi la mi- tad de los cambios en los sistemas vivos". En J. Wagensberg y J. Agusti (eds.), El progreso, Barcelona, Tusquets.

Newton, I. (1997, 1687): Principios matemáticos de la filosofía natural, Madrid, Altaya.

Tansley, A. (1935): "The Use and Abuse of Vegetal Concepts and Terms", en Ecology, 16, 284-307.

Walter, L. (2005) "Margalef y la sucesión ecológica", en Ecosistemas, 14 (1) (URL: http://www.revistaecosistemas. net/articulo.asp?ld=70).

Zubiri, X. (2006): Tres dimensiones del ser humano: Individual, Social, Histórica, Madrid, Alianza.

- (2008): Espacio, Tiempo, Materia. Nueva edición, Madrid, Alianza. 